\title{
Evaluation of Serum Ferritin as a Prognostic Marker in Acute Hemorrhagic Stroke
}

\author{
Sowri Rajan Rajendran ${ }^{1}$ Sivakumar Periyasamy ${ }^{1}$ Moonishaa Thiyagarajan Manjuladevi ${ }^{2}$ Neethu George ${ }^{3}$ \\ ${ }^{1}$ Department of General Medicine, Trichy SRM Medical College \\ Hospital \& Research Centre, (Affiliated to The Tamil Nadu \\ Dr. M.G.R. Medical University), Irungalur, Trichy, Tamil Nadu, India \\ 2Department of Biochemistry, Trichy SRM Medical College Hospital

\begin{abstract}
Address for correspondence Sivakumar Periyasamy, MD, Department of General Medicine, Trichy SRM Medical College Hospital \& Research Centre, (Affiliated to The Tamil Nadu Dr. M.G.R. Medical University), Irungalur, Trichy 621105, Tamil Nadu, India (e-mail: moonishaatm89@gmail.com).
\end{abstract}

\& Research Centre, (Affiliated to The Tamil Nadu Dr. M.G.R.

Medical University), Irungalur, Trichy, Tamil Nadu, India

${ }^{3}$ Department of Community Medicine, Dhanalakshmi Srinivasan

Medical College and Hospital (Affiliated to The Tamil Nadu Dr. M.G.R.

Medical University), Siruvachur, Perambalur, Tamil Nadu, India

J Neurosci Rural Pract 2020;11:72-77

\begin{abstract}
Keywords

- serum ferritin

- acute hemorrhagic stroke

- prognosis

- modified Rankin scale

Background Acute hemorrhagic stroke (AHS) resulting from intracerebral hemorrhage $(\mathrm{ICH})$ is a rampant neurological disorder with devastating consequences, particularly in Indians. Recently, serum ferritin levels have been related to adverse cardiovascular and stroke outcomes. We aimed to evaluate the prognostic utility of serum ferritin in AHS.

Materials and Methods Admission serum ferritin levels were estimated in 50 AHS patients with primary supratentorial hemorrhage. Study subjects were categorized based on their prognostic scores in modified Rankin scale (mRS) assessment. Ferritin levels were compared across the study groups, correlated with mRS and other ICH severity indicators.

Results Serum ferritin and other ICH severity indices such as Glasgow coma scale (GCS) and ICH volume were significantly altered in the mRS groups by the end of 7th and 30th days of hospitalization. Elevated ferritin levels, ICH volume together with decreased GCS, characterized the groups with adverse prognosis. Serum ferritin moderately correlated with GCS $(r=-0.643)$, ICH volume $(r=0.562)$, and had significantly higher correlations with long-term prognostic scores of 7 th day mRS $(r=0.802)$ and 30th day mRS $(r=0.916)$.

Conclusion Elevated admission serum ferritin levels indicate poor AHS short-term and long-term outcomes, thereby making serum ferritin a possible prognostic index for the same.
\end{abstract}

\section{Introduction}

Stroke or cerebrovascular accident characterized by acute neurological deficit can be of ischemic or hemorrhagic origin. Besides being a global leading cause of death next only to ischemic heart disease, stroke is a predominant cause of adult disability. ${ }^{1}$ People of South Asia and the developing countries have greater stroke risks, thus accounting for the higher prevalence of approximately 44.29 to 559/100,000 and cumulative incidence of 105 to $152 / 100,000$ persons per year across India. ${ }^{2}$ Among the subtypes, acute hemorrhagic stroke (AHS) is less common (approximately 13\% of all stroke cases) but more severe resulting in $40 \%$ of deaths due to stroke. Comparatively, AHS is more frequent in Indians accounting for nearly 20 to $32 \%$ of all stroke cases. $^{3}$

Among the hemorrhagic stroke types, intracerebral hemorrhage (ICH) is more common than subarachnoid
License terms

(요 (1) $\odot \circledast$ 
hemorrhage. Hypertension is the predominant cause of primary ICH. Besides clinical diagnosis, radio imaging, especially computed tomography (CT) of the brain, is indispensable for diagnosis as well as prognosis of ICH. Radiological signs, such as size, site, extent of ICH, surrounding edema, and hemorrhage extension, and clinical signs, such as loss of consciousness measured by Glasgow coma scale (GCS) and blood pressure (BP) indices, are some of the early prognosticators of $\mathrm{ICH}^{4}{ }^{4}$ The long-term stroke outcome and functional disability can be systematically ascertained by means of modified Rankin scale (mRS), which is an extensively validated stroke scoring system. ${ }^{5}$

Ferritin, a protein serving as body iron reservoir, stores excess iron, thereby preventing toxic effects of free radicals generated by unbound iron. Serum ferritin levels indicating body iron stores are routinely used for laboratory diagnosis of iron deficiency and overload conditions. In the recent past, several researchers studied the possible role of serum ferritin in predicting the iron-mediated free radical injury in the pathogenesis of cardiovascular diseases. Salonen et al and van der A et al concluded that elevated serum ferritin levels were associated with higher risks of acute myocardial infarction in Finnish men and higher risks of ischemic stroke in Dutch postmenopausal women, respectively. ${ }^{6,7}$ A study by Dávalos et al ascertained serum ferritin's adverse prognostic role in ischemic stroke. ${ }^{8}$ A study in Spanish ICH patients revealed independent association of high admission serum ferritin levels to unfavorable outcomes. ${ }^{9}$ Hence, the present study aimed at evaluating the prognostic utility of serum ferritin in stroke due to ICH in Indian population.

\section{Materials and Methods}

This cross-sectional study conducted at a tertiary care teaching hospital was approved by the Institutional Ethics Committee. Fifty consecutive patients admitted for their first stroke episode, who were diagnosed clinically and radiologically as primary supratentorial hemorrhage, were included in the study. Cases of secondary ICH, ischemic stroke, anemia, severe alcohol consumption, chronic liver disease, chronic kidney disease, and hematological malignancies were excluded.

On admission, all subjects underwent a series of study evaluation such as detailed case history, clinical examination, CT scan of the brain, routine blood investigations (complete blood count, random blood sugar, and renal and liver function tests), and serum ferritin estimation; later during the hospital stay, they were followed up for prognostic assessment. Case history included stroke symptomatology such as headache, vomiting, loss of consciousness, seizures, focal neurological deficit (FND), and past history of hypertension, diabetes mellitus, and drug intake. Clinical examination comprised recording the vital signs, GCS on admission, systemic examination findings, signs of neurological deficit, and raised intracranial tension.

Soon after admission, all stroke patients were subjected to nonenhanced CT scan of the brain and the subjects with primary ICH located at supratentorial region were selected for this study. Important brain CT findings such as size, site, intraventricular extension of hematoma, and midline shift if any were noted. The hematoma volume was calculated from the index slice (slice of CT of the brain showing largest image of hematoma) by applying the much validated Ellipsoid formula as follows: ICH volume (in $\mathrm{mL})=\left(\begin{array}{lll}A & B & C\end{array}\right) / 2$, where $\mathrm{A}($ in $\mathrm{cm})=$ largest hematoma diameter, $\mathrm{B}($ in $\mathrm{cm})=$ hematoma diameter at 90 degrees to $\mathrm{A}$, and $\mathrm{C}=$ approximate number of 10 -mm slices with hematoma. ${ }^{10}$

For serum ferritin estimation, $2 \mathrm{~mL}$ of venous blood samples were collected from all subjects within 72 hours of symptom onset. Serum ferritin was estimated by a classic sandwich enzyme-linked immunosorbent assay method involving immunoenzymometric sequential assay type 4 , using AccuBind serum ferritin kit manufactured by Monobind Inc., United States. Adult reference range for serum ferritin levels are 20 to $250 \mathrm{ng} / \mathrm{mL}$ in males and 10 to $120 \mathrm{ng} / \mathrm{mL}$ in females. ${ }^{11}$

Prognostic assessments conducted at 7th and 30th days of hospitalization involved recording of $\mathrm{mRS}$ scores for the subjects, ranging from 0 (perfect health) to 6 (death) depending on the severity of functional disability poststroke., ${ }^{5,12}$ Based on their mRS scores, the study subjects were categorized into three groups as follows: subjects with good prognosis $(\mathrm{mRS}=0-2)$, bad prognosis ( $\mathrm{mRS}=3-5)$, and worst prognostic event of death $(\mathrm{mRS}=6)$. Ferritin levels were compared across the study groups, and also correlated with ICH severity indices.

\section{Statistics}

The data were entered in Microsoft excel and analyzed using statistical software SPSS 20.0. Descriptive details were presented as frequencies, means, medians, interquartile range, and standard deviations. Inferential statistical methods such as one-way analysis of variance and Kruskal-Wallis tests were used to compare serum ferritin and other parameters among the study groups, while Spearman's correlation was used to determine any significant associations of serum ferritin levels with ICH severity indices. A p-value of less than 0.05 was considered as significant.

\section{Results}

The study population comprised 39 (78\%) males and 11 (22\%) females, with the mean age of $55.82 \pm 12.67$ years. Frequencies of FND and other classical ICH symptoms were as expected ( $\boldsymbol{\sim}$ Table $\mathbf{1}$ ). Data on history for ICH risk factors revealed that $9(18 \%)$ subjects had diabetes mellitus and almost 49 (98\%) of them had systemic hypertension, which led to the elevated BP indices in most of the participants (-Table 1). Examination of cardiovascular and respiratory systems was normal in all subjects.

Neurological examination results exposed a median GCS of $8(6,10)$, with hemiparesis and normal fundus in all the participants. Left-sided hemiparesis was present in 24 (48\%) of them, while 26 (52\%) had right-sided hemiparesis. CT of the brain reports disclosed that the commonest site for ICH 
was the gangliocapsular region, with a median ICH volume of $57(36,84.5) \mathrm{mL}$, and only $1(2 \%)$ patient had midline shift, while 12 (24\%) of them had intraventricular hematoma extension (-Table 1). The median serum ferritin level was $269.5(186.75,367.5) \mathrm{ng} / \mathrm{mL}$ in the entire study group.

At the end of first week of hospitalization, the subjects $(n=50)$ were distributed into three $\mathrm{mRS}$ groups as follows: 8 (16\%), 33 (66\%), and 9 (18\%) subjects came under the good prognosis, bad prognosis, and death groups, respectively ( $\mathbf{- F i g . ~ 1 ) . ~ A m o n g ~ t h e ~ s t u d y ~ g r o u p s , ~ h i g h e r ~ l e v e l s ~ o f ~ B P ~}$ indices such as systolic blood pressure (SBP), diastolic blood pressure (DBP), pulse pressure (PP), and mean arterial pressure (MAP) were present in the adverse prognostic groups,

Table 1 Clinical data of the study population $(n=50)$

\begin{tabular}{|l|l|}
\hline History of presenting complaints & Frequency (\%) \\
\hline Focal neurological deficit & $50(100 \%)$ \\
\hline Headache & $25(50 \%)$ \\
\hline Vomiting & $35(70 \%)$ \\
\hline Loss of consciousness & $10(20 \%)$ \\
\hline Seizures & $5(10 \%)$ \\
\hline Clinical examination of vital signs & Mean \pm SD \\
\hline Pulse rate (per min) & $71.6 \pm 10.89$ \\
\hline Systolic blood pressure $(\mathrm{mm} \mathrm{Hg})$ & $164 \pm 14.99$ \\
\hline Diastolic blood pressure $(\mathrm{mm} \mathrm{Hg})$ & $95.92 \pm 9.03$ \\
\hline Pulse pressure (mm Hg) & $68.72 \pm 13.9$ \\
\hline Mean arterial pressure $(\mathrm{mm} \mathrm{Hg})$ & $118.83 \pm 9.23$ \\
\hline CT of the brain-ICH location & Frequency (\%) \\
\hline Gangliocapsular & $35(70 \%)$ \\
\hline Thalamic & $7(14 \%)$ \\
\hline Lobar & $8(16 \%)$ \\
\hline
\end{tabular}

Abbreviations: $\mathrm{CT}$, computed tomography; ICH, intracerebral hemorrhage; $\mathrm{SD}$, standard deviation. but the increases were not significant. However, subjects with significantly higher mean age had bad prognosis or died within a week of hospitalization compared with their counterparts with good prognostic mRS scores $(p<0.05)$ (-Table 2). Also, there were significant decline of GCS scores, increase in ICH volume, and elevation of serum ferritin levels in the adverse prognostic groups than those with good prognosis $(p<0.05)(-$ Table 2$)$.

The survivors $(n=41)$ were followed up till 30 th day of hospitalization for re-evaluation of prognostic mRS scores, so that $8(19 \%), 27$ (66\%), and 6 (15\%) of them, respectively, came under the good prognosis, bad prognosis, and death groups ( - Fig. 1). Only ICH severity indices such as GCS, ICH volume, and serum ferritin were significantly altered across the groups; adverse prognostic groups showed decreasing GCS with raising ICH volume and serum ferritin levels $(p<0.05)$ ( - Table 3 ).

Spearman's correlation analysis concluded moderate associations of serum ferritin with other ICH severity indices such as GCS $(r=-0.643, p<0.01)$ and ICH volume $(r=0.562, p<0.01)$. Correlations of these parameters with long-term stroke prognosis (7th and 30th days mRS scores) inferred significantly higher correlations of serum ferritin with 7th day mRS $(r=0.802, p<0.01)$ and 30th day mRS scores $(r=0.916, p<0.01)$ (-Table 4).

\section{Discussion}

The demographic results of our study depicted a strikingly higher incidence of primary ICH in males (78\%) with a massive sex gap, and moreover, the mean age is also very much lowered ( 55.82 years). These results are akin to the global burden of disease study findings of early stroke onset in South Asians particularly men, due to their prevalent stroke risk factors. ${ }^{13,14}$ Case history data displayed the classical ICH manifestations such as FND and signs of raised intracranial pressure such as headache and vomiting.

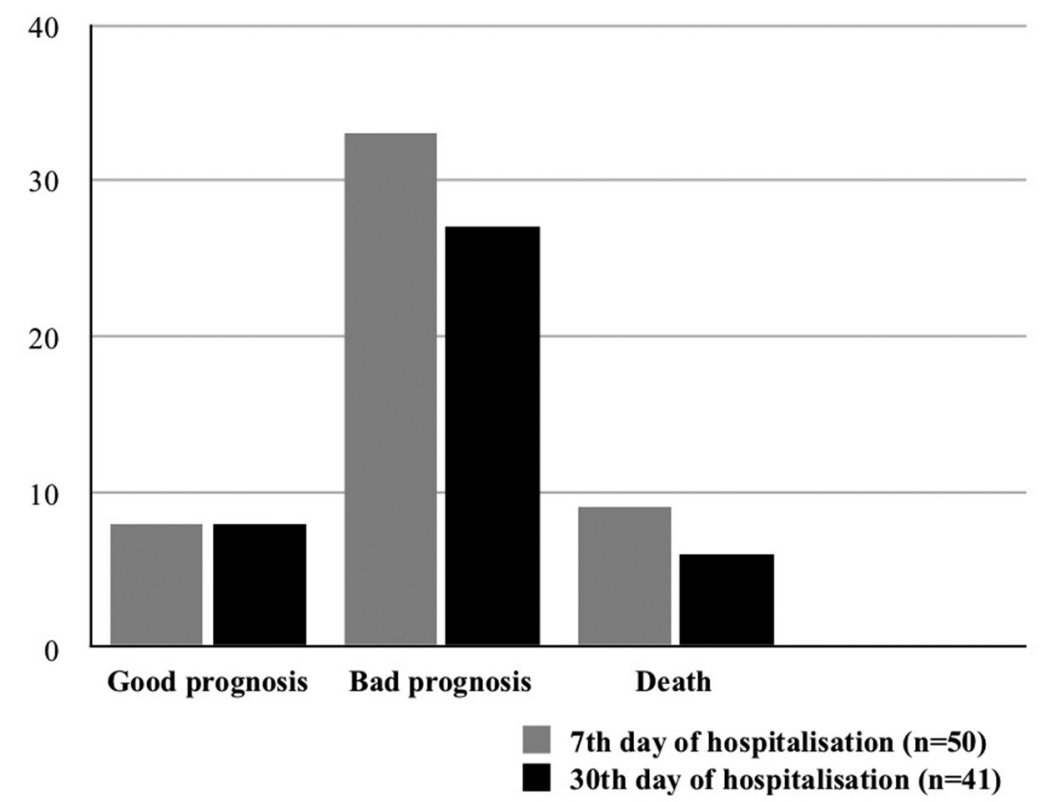

Fig. 1 Distribution of study groups on 7th and 30th days of hospitalization. Based on mRS scores, the subjects were categorized into three groups as follows: good prognosis (mRS $=0-2)$, bad prognosis $(m R S=3-5)$, and death $(m R S=6)$. mRS, modified Rankin scale. 
Table 2 Comparison of parameters across study groups on 7th day of hospitalization

\begin{tabular}{|c|c|c|c|c|c|}
\hline Parameter & $\begin{array}{l}\text { Good prognosis (7th day } \\
\text { mRS }=0-2)(n=8)\end{array}$ & $\begin{array}{l}\text { Bad prognosis (7th day } \\
\mathrm{mRS}=3-5)(n=33)\end{array}$ & $\begin{array}{l}\text { Death (7th day } \\
\mathrm{mRS}=6)(n=9)\end{array}$ & $\begin{array}{l}\text { Table } \\
\text { value }\end{array}$ & $p$-Value \\
\hline Age $(y)$ & $44.88 \pm 11.76$ & $56.18 \pm 12.23$ & $64.22 \pm 7.98$ & 5.996 & $0.005^{a, b}$ \\
\hline $\mathrm{SBP}(\mathrm{mm} \mathrm{Hg})$ & $162.25 \pm 16.61$ & $163.52 \pm 14.23$ & $170.89 \pm 16.43$ & 0.976 & $0.384^{\mathrm{a}}$ \\
\hline $\mathrm{DBP}(\mathrm{mm} \mathrm{Hg})$ & $95 \pm 8.35$ & $95.58 \pm 10.02$ & $98 \pm 5.48$ & 0.296 & $0.745^{\mathrm{a}}$ \\
\hline $\mathrm{PP}(\mathrm{mm} \mathrm{Hg})$ & $67.25 \pm 14.30$ & $67.94 \pm 13.69$ & $72.89 \pm 15.40$ & 0.488 & $0.617^{a}$ \\
\hline MAP $(\mathrm{mm} \mathrm{Hg})$ & $117.42 \pm 9.64$ & $118.22 \pm 9.63$ & $122.30 \pm 7.57$ & 0.785 & $0.462^{\mathrm{a}}$ \\
\hline $\operatorname{GCS}(x / 15)$ & $12(11.25,12.75)$ & $8(7,9.5)$ & $5(3.5,7.5)$ & 7.368 & $0.008^{b, c}$ \\
\hline $\begin{array}{l}\text { ICH volume } \\
(\mathrm{mL})\end{array}$ & $24(16.25,32)$ & $58(41.5,79)$ & $86(78,93)$ & 8.193 & $0.002^{\mathrm{b}, \mathrm{c}}$ \\
\hline $\begin{array}{l}\text { Serum ferritin } \\
(\mathrm{ng} / \mathrm{mL})\end{array}$ & $121(79,136)$ & $270(212,370)$ & $366(306,399)$ & 8.571 & $0.002^{\mathrm{b}, \mathrm{c}}$ \\
\hline
\end{tabular}

Abbreviations: DBP, diastolic blood pressure; GCS, Glasgow coma scale; ICH, intracerebral hemorrhage; MAP, mean arterial pressure; mRS, modified Rankin scale; PP, pulse pressure; SBP, systolic blood pressure.

ane-way analysis of variance.

'Significant $p$-value $<0.05$.

'Kruskal-Wallis' test.

Table 3 Comparison of parameters across study groups on 30th day of hospitalization

\begin{tabular}{|c|c|c|c|c|c|}
\hline Parameter & $\begin{array}{l}\text { Good prognosis (30th day } \\
\mathrm{mRS}=0-2)(n=8)\end{array}$ & $\begin{array}{l}\text { Bad prognosis (30th day } \\
\text { mRS }=3-5)(n=27)\end{array}$ & $\begin{array}{l}\text { Death (30th day } \\
\text { mRS = 6) }(n=6)\end{array}$ & $\begin{array}{l}\text { Table } \\
\text { value }\end{array}$ & $p$-Value \\
\hline Age (y) & $44.88 \pm 11.76$ & $56.41 \pm 11.89$ & $55.17 \pm 14.84$ & 2.747 & $0.077^{a}$ \\
\hline $\mathrm{SBP}(\mathrm{mm} \mathrm{Hg})$ & $162.25 \pm 16.61$ & $160.96 \pm 13.31$ & $175 \pm 13.49$ & 2.495 & $0.096^{\mathrm{a}}$ \\
\hline $\mathrm{DBP}(\mathrm{mm} \mathrm{Hg})$ & $95 \pm 8.35$ & $95.48 \pm 10.13$ & $96 \pm 10.43$ & 0.018 & $0.982^{\mathrm{a}}$ \\
\hline $\mathrm{PP}(\mathrm{mm} \mathrm{Hg})$ & $67.25 \pm 14.30$ & $65.48 \pm 13.68$ & $79 \pm 6.78$ & 2.619 & $0.086^{\mathrm{a}}$ \\
\hline MAP (mm Hg) & $117.42 \pm 9.64$ & $117.31 \pm 9.26$ & $122.33 \pm 11.09$ & 0.696 & $0.505^{\mathrm{a}}$ \\
\hline $\operatorname{GCS}(x / 15)$ & $12(11.25,12.75)$ & $8(7,10)$ & $5.5(4.75,7.25)$ & 23.363 & $<0.001^{c}$ \\
\hline $\begin{array}{l}\text { ICH volume } \\
(\mathrm{mL})\end{array}$ & $24(16.25,32)$ & $56(40,78)$ & $66(51,87.5)$ & 15.164 & $0.001^{b, c}$ \\
\hline $\begin{array}{l}\text { Serum ferritin } \\
(\mathrm{ng} / \mathrm{mL})\end{array}$ & $121(79,136)$ & $260(196,296)$ & $390(346,403)$ & 23.561 & $<0.001^{c}$ \\
\hline
\end{tabular}

Abbreviations: DBP, diastolic blood pressure; GCS, Glasgow coma scale; ICH, intracerebral hemorrhage; MAP, mean arterial pressure; mRS, modified Rankin scale; PP, pulse pressure; SBP, systolic blood pressure.

aOne-way analysis of variance.

'Kruskal-Wallis' test.

'Significant $p$-value $<0.05$.

Pertaining to ICH risk factors, as in most other studies, systemic hypertension emerged as the most predominant risk factor in our study subjects, thus leading to their elevated levels of BP indices (SBP, DBP, PP, and MAP) ${ }^{15}$ Neurological examination showed reduced GCS score (median GCS $=8$ ) with unilateral hemiparesis. CT of the brain reports revealed higher ICH volume (median volume $=57 \mathrm{~mL}$ ) with most hematomas $(84 \%)$ confined to the gangliothalamic region. These clinical and radiological findings are comparable with previous study results. ${ }^{16,17}$

By 7th day of hospitalization, majority ( $n=42$ [84\%]) of subjects fell into either of the adverse prognostic groups having $\mathrm{mRS}$ scores $>2$ and increasing levels of BP indices (SBP, DBP, PP, and MAP) that were not significantly different from the good prognostic group. This is in contrary to studies showing associations of higher BP indices with poor ICH outcomes, thereby recommending early stringent BP control for better patient outcomes. ${ }^{18}$ But our results are comparable to those of Qureshi et al showing no significant associations between BP alterations and ICH outcomes. ${ }^{19}$

As mRS reassessment was performed on 30th day of hospitalization, again most survivors $(n=33$ [81\%]) with mRS scores $>2$ came into the two adverse prognosis groups. Although mean age was significantly higher in the adverse $\mathrm{mRS}$ groups at 7 th day of hospitalization, there was no such marked age variations between the mRS groups at 30th day of hospitalization. So, we presume that advancing age could predict only the short-term detrimental outcomes of $\mathrm{ICH}$ and has no value in long-term AHS prognosis. The probable reason for this could be the early AHS onset in our study subjects due to their ethnic predisposition, and these findings were identical to those of Hegde and Menon..$^{13,20}$

When rest of the parameters were compared across the mRS groups at 7th and 30th days of hospitalization, significant alterations were deductible only with respect to ICH prognosticators such as GCS scores, ICH volume, and serum 
Table 4 Correlation of serum ferritin and other ICH severity parameters with mRS scores

\begin{tabular}{|c|c|c|}
\hline & $\begin{array}{l}\text { Correlation } \\
\text { coefficient }\end{array}$ & $p$-Value ${ }^{a}$ \\
\hline \multicolumn{3}{|c|}{ Parameters correlated with 7th day mRS } \\
\hline $\operatorname{GCS}(x / 15)$ & -0.740 & $<0.01$ \\
\hline $\mathrm{ICH}$ volume (mL) & 0.681 & $<0.01$ \\
\hline Serum ferritin (ng/mL) & 0.802 & $<0.01$ \\
\hline \multicolumn{3}{|c|}{ Parameters correlated with 30th day mRS } \\
\hline GCS $(x / 15)$ & -0.739 & $<0.01$ \\
\hline $\mathrm{ICH}$ volume $(\mathrm{mL})$ & 0.582 & $<0.01$ \\
\hline Serum ferritin (ng/mL) & 0.916 & $<0.01$ \\
\hline
\end{tabular}

Abbreviations: GCS, Glasgow coma scale; $\mathrm{ICH}$, intracerebral hemorrhage; mRS, modified Rankin scale.

aSpearman's correlation.

ferritin levels. Likewise, studies by Safatli et al and Broderick et al inferred that decreased admission GCS scores together with raised ICH volume are strong predictors of 30-day ICH outcome. ${ }^{17,21}$ Thus, admission GCS scores and ICH volume are good performers among the existing ICH prognosticators.

Serum ferritin levels remained significantly elevated in the study groups with poor prognosis at 7th and until 30th days of hospitalization. Thereupon, admission serum ferritin levels could become a long-term ICH prognostic index similar to GCS scores and ICH volume, as rightly claimed by Pérez de la Ossa et al. ${ }^{9}$ Among the three prognostic indices, admission serum ferritin levels exhibited significantly higher correlations with 7th day mRS scores $(r=0.802)$ and 30th day mRS scores $(r=0.916)$, thus outperforming both admission GCS scores and $\mathrm{ICH}$ volume to emerge as the best long-term ICH prognostic index.

Ferritin, being an acute phase protein, should be estimated within 72 hours of symptom onset to eliminate any possible elevations in an acute phase reaction. The same was endorsed by Armengou et al concluding that unlike other acute phase reactants, serum ferritin levels were not altered before 72 hours following stroke onset. ${ }^{22}$ So, any early alterations in its levels are not due to acute phase response but could be consequent to the pathologic progression of the hemorrhagic lesion.

The underlying mechanisms postulated for the adverse prognostic role of ferritin are local iron overload at hematoma site and the resultant iron-mediated neurotoxicity. Iron-mediated free radical generation and oxidative damage lead to neuronal injury and perihematomal edema formation in cases of cerebral hemorrhage. ${ }^{23,24}$ Serum ferritin can quantify the body iron stores more reliably than other iron indices such as serum iron, transferrin, total iron-binding capacity as the latter ones carried greater biological variabilities than ferritin. ${ }^{25}$

Xie et al established serum ferritin to be an independent predictor of long-term functional outcome in neurocritically ill patients amidst all other iron indices. ${ }^{26}$ There are also evidences validating that decreasing body iron by injecting iron chelators such as deferoxamine could alleviate the neuropathological changes of ICH in experimental animal models..$^{27,28}$ As elevated admission serum ferritin is associated with adverse ICH outcomes in the present study, the advantages of hypoferremia and iron lowering therapeutic targets in ICH patients could be the domains of future research.

\section{Conclusion}

Elevated admission serum ferritin levels indicate poor AHS outcomes on short-term and long-term. Hence serum ferritin is a possible prognostic index for AHS due to primary supratentorial hemorrhage.

\section{Funding}

None.

\section{Conflict of Interest}

None declared.

\section{References}

1 Lozano R, Naghavi M, Foreman K, et al. Global and regional mortality from 235 causes of death for 20 age groups in 1990 and 2010: a systematic analysis for the Global Burden of Disease Study 2010. Lancet 2012;380(9859):2095-2128

2 Kamalakannan S, Gudlavalleti ASV, Gudlavalleti VSM, Goenka S, Kuper H. Incidence \& prevalence of stroke in India: a systematic review. Indian J Med Res 2017;146(2):175-185

3 Banerjee TK, Das SK. Fifty years of stroke researches in India. Ann Indian Acad Neurol 2016;19(1):1-8

4 Rådberg JA, Olsson JE, Rådberg CT. Prognostic parameters in spontaneous intracerebral hematomas with special reference to anticoagulant treatment. Stroke 1991;22(5):571-576

5 van Swieten JC, Koudstaal PJ, Visser MC, Schouten HJ, van Gijn J. Interobserver agreement for the assessment of handicap in stroke patients. Stroke 1988;19(5):604-607

6 Salonen JT, Nyyssönen K, Korpela H, Tuomilehto J, Seppänen $\mathrm{R}$, Salonen R. High stored iron levels are associated with excess risk of myocardial infarction in eastern Finnish men. Circulation 1992;86(3):803-811

7 van der A DL, Grobbee DE, Roest M, Marx JJ, Voorbij HA, van der Schouw YT. Serum ferritin is a risk factor for stroke in postmenopausal women. Stroke 2005;36(8):1637-1641

8 Dávalos A, Fernandez-Real JM, Ricart W, et al. Iron-related damage in acute ischemic stroke. Stroke 1994;25(8):1543-1546

9 Pérez de la Ossa N, Sobrino T, Silva Y, et al. Iron-related brain damage in patients with intracerebral hemorrhage. Stroke 2010;41(4):810-813

10 KothariRU, BrottT, BroderickJP, etal. The ABCs of measuring intracerebral hemorrhage volumes. Stroke 1996;27(8):1304-1305

$11 \mathrm{Wu} \mathrm{A}$, Tietz Clinical Guide to Laboratory Tests, 4th ed. Missouri: Saunders; 2009:392

12 Banks JL, Marotta CA. Outcomes validity and reliability of the modified Rankin scale: implications for stroke clinical trials: a literature review and synthesis. Stroke 2007;38(3):1091-1096

13 Mehndiratta MM, Khan M, Mehndiratta P, Wasay M. Stroke in Asia: geographical variations and temporal trends. J Neurol Neurosurg Psychiatry 2014;85(12):1308-1312

14 Barker-Collo S, Bennett DA, Krishnamurthi RV, et al. GBD 2013 Writing GroupGBD 2013 Stroke Panel Experts Group. Sex differences in stroke incidence, prevalence, mortality and 
disability-adjusted life years: results from the Global Burden of Disease Study 2013. Neuroepidemiology 2015;45(3):203-214

15 Sturgeon JD, Folsom AR, Longstreth WT Jr, Shahar E, Rosamond WD, Cushman M. Risk factors for intracerebral hemorrhage in a pooled prospective study. Stroke 2007;38(10):2718-2725

16 Ojaghihaghighi S, Vahdati SS, Mikaeilpour A, Ramouz A. Comparison of neurological clinical manifestation in patients with hemorrhagic and ischemic stroke. World J Emerg Med 2017;8(1):34-38

17 Safatli DA, Günther A, Schlattmann P, Schwarz F, Kalff R, Ewald C. Predictors of 30-day mortality in patients with spontaneous primary intracerebral hemorrhage. Surg Neurol Int 2016;7(Suppl 18):S510-S517

18 Anderson CS, Heeley E, Huang Y, et al. INTERACT2 Investigators. Rapid blood-pressure lowering in patients with acute intracerebral hemorrhage. N Engl J Med 2013;368(25):2355-2365

19 Qureshi AI, Palesch YY, Martin R, et al. Antihypertensive Treatment of Acute Cerebral Hemorrhage Study Investigators. Effect of systolic blood pressure reduction on hematoma expansion, perihematomal edema, and 3-month outcome among patients with intracerebral hemorrhage: results from the antihypertensive treatment of acute cerebral hemorrhage study. Arch Neurol 2010;67(5):570-576

20 Hegde A, Menon G. Modifying the intracerebral hemorrhage score to suit the needs of the developing world. Ann Indian Acad Neurol 2018;21(4):270-274
21 Broderick JP, Brott TG, Duldner JE, Tomsick T, Huster G. Volume of intracerebral hemorrhage. A powerful and easy-to-use predictor of 30-day mortality. Stroke 1993;24(7):987-993

22 Armengou A, Dávalos A, Fernandez-Real JM, Castillo J. Serum ferritin concentrations are not modified in the acute phase of ischemic stroke. Stroke 1998;29(1):258-260

23 Lou M, Lieb K, Selim M. The relationship between hematoma iron content and perihematoma edema: an MRI study. Cerebrovasc Dis 2009;27(3):266-271

24 Mehdiratta M, Kumar S, Hackney D, Schlaug G, Selim M. Association between serum ferritin level and perihematoma edema volume in patients with spontaneous intracerebral hemorrhage. Stroke 2008;39(4):1165-1170

25 Pfeiffer CM, Looker AC. Laboratory methodologies for indicators of iron status: strengths, limitations, and analytical challenges. Am J Clin Nutr 2017;106(Suppl 6):1606S-1614S

26 Xie L, Peng Y, Huang K, Wu Y, Wang S. Predictive value of iron parameters in neurocritically ill patients. Brain Behav 2018;8(12):e01163

27 Okauchi M, Hua Y, Keep RF, Morgenstern LB, Schallert T, Xi G. Deferoxamine treatment for intracerebral hemorrhage in aged rats: therapeutic time window and optimal duration. Stroke 2010;41(2):375-382

28 Gu Y, Hua Y, Keep RF, Morgenstern LB, Xi G. Deferoxamine reduces intracerebral hematoma-induced iron accumulation and neuronal death in piglets. Stroke 2009;40(6):2241-2243 